\title{
Evaluation of Serum Level of Claudin- 3 and Its Association with Disease Severity in Patients with Psoriasis in Iraqi patients.
}

Yasser N. Ahmed ${ }^{1}$, Ghassan A. Al-Shamma ${ }^{2}$ and Abdullah Salih Hassen

${ }^{1 *}$ College of Medicine, University of Fallujah, Iraq

${ }^{2}$ College of Medicine, Al-Nahrain University, Iraq

${ }^{3}$ College of Medicine, University of Anbar, Iraq

*Corresponding Author : cas77nij@gmail.com

\begin{abstract}
:
Background Psoriasis is a prevalent inflammatory skin disease. Psoriasis is a complex illness in which environmental variables acting on individuals with unique genetic predisposition causing immunological dysregulation. Claudins are transmembrane proteins that help to generate tight junctions by binding to the actin cytoskeleton. Claudin 3 in the blood is thought to be a good indicator of intestinal permeability.

Objective: The aim of this study was to detect of the alteration of claudin-3 in psoriasis patient and find the correlation between severity and concentration of the claudin-3.

Patients and methods: forty psoriatic patients (25males and 15 females) and thirty normal healthy controls (19 men and 11 females) who were age and sex matched to the cases group were included in this study. They were chosen at random from Al Fallujah hospital Dermatology Department outpatient clinic.

Result: When compared to the control group, the psoriasis group had substantially greater levels of claudin-3 (mean=2.18 \pm 0.16 versus $1.27 \pm 0.03$; p0.0001). Furthermore, the amount of claudin-3 rose progressively as the severity grade increased (001). There were no significant correlations between claudin-3 levels and gender, dietary status, or family history in the psoriasis group ( $>0.05$ for each).
\end{abstract}

Conclusion: Claudin-3 levels were considerably greater in psoriasis patients than in healthy controls. PASI levels were shown to be linked to claudin-3 levels.

\section{Keywords: Claudin- 3, PASI score, Psoriasis, hyperlipidemia, hyperuricemia.}

\section{Introduction:}

Psoriasis is a multifactorial illness in which certain environmental variables act on people who have a certain genetic predisposition causing immunological dysregulation and aberrant keratinization, resulting in the formation of characteristic cutaneous lesions [1].

It is a genetically based chronic inflammatory skin disease characterised by epidermal hyperproliferation, aberrant keratinocyte differentiation, T-lymphocyte infiltration, and elevated cytokine production, resulting in the development of inflamed plaques [2].

Psoriasis is characterised histologically by epidermal hyperproliferation with aberrant keratinocyte differentiation, as well as dermal inflammatory cells [3].

Claudins (CLDN) are tetraspan proteins that have at least 24 members. Claudin-3 has been demonstrated to be expressed more strongly in distal than proximal tubular organs such as the gut and nephron. It has been 
demonstrated that the expression of claudin-3 along rat intestinal segments corresponds with barrier characteristics[4] .

Recently, there has been a lot of attention on the connection between the gut microbiota, the intestinal barrier, and the immune system. The so-called gut skin axis has been identified as a critical component in the Psoriasis pathogenesis and possible therapeutic targets[5]. The function of the intestinal epithelial barrier consists of numerous defensive mechanisms that may be classified into physical and immunological barriers[6]. The physical intestinal barrier is made up of an epithelial cell lining that is linked by tight junctions[7]. Tight junctions are held in place by the cytoskeleton of filamentous actin (F-actin) [8] . Zonula occludins proteins (ZO-1, ZO-2, and ZO-3) are intracellular tight junction proteins that connect the cell cytoskeleton to transmembrane tight junction proteins such as claudins, occludins, and junctional adhesion molecules (JAM). Whereas occludin and JAM have a regulatory role, claudins are transmembrane proteins that are primarily important for the function of the intestinal barrier [9] . The absence of certain claudins results in a lack of intestinal barrier function. Claudin-3 in the blood is thought to be a good indicator of intestinal permeability [10]. The gastrointestinal tract's physiological colonisation supports intestinal barrier development, whereas dysbiosis disturbs this process and contributes to increased gut permeability. The introduction of bacteria, microbial toxins, and metabolites into the peripheral circulation activates the immune system [11]. Disruptions in the intestinal barrier have been linked to metabolic and inflammatory psoriatic comorbidities. As a result, the amount of claudin 3 may be a therapeutically relevant measure for gut permeability, which is disrupted in psoriasis. Furthermore, strengthening the intestinal barrier may be a novel therapeutic focus in psoriasis [12] .

The current study was conducted to compare blood levels of claudin-3 in psoriasis patients to a control group and to connect these levels with disease severity.

\section{Materials and methods}

This descriptive and interventional study was conducted from March 2021 until the end of July 2021 at the Department of Biochemistry, College of Medicine, University of Al Nahrain, and at the Center of Dermatology and Venereology / AL Fallujah hospital. It includes 62 patients diagnosed with psoriasis by a Consultant Dermatologist. They ranged in age from 14 to 64 years old and had varying disease durations. Twelve of these patients were eliminated from the trial because some of them had a psoriasis area severity index (PASI) score of less than (7.5) and others had inflammatory disease other than or in addition to psoriasis. The task was divided into two groups, all of which were equal in age and sex.

\section{Inclusion criteria:}

Included patients 14 years and older who were on Inclusion of newly diagnosed group for assessing the effect of psoriasis treatment or biomarker levels

\section{Exclusion criteria:}

Included patients with a history of cancer or who were taking medicine for other inflammatory and autoimmune diseases, dermatological problems, diabetes, chronic liver diseases, or chronic kidney illnesses.

A detailed history is taken, including age, gender, employment, marital status, particular habits, nutritional intake, related psychiatric disturbance, associated medical or surgical disorders, and drug use.

\section{All patients underwent the following:}


Detailed general examination: all important clinical data, such as age, gender, body mass index (BMI), Smoking, Disease duration (years) and PASI score were recorded

All patients had their lipid profile and serum claudin-3 levels tested in the lab: $5 \mathrm{ml}$ morning venous blood samples were obtained from each participant involved in this trial following an overnight $12 \mathrm{~h}$ fast. After centrifuging the blood, the sera were separated for analysis. ELISA method was used to assess serum claudin-3 levels while the lipid profile was determined by using Enzymatic spectrophotometric methods utilizing a Spinreact laboratory kit.

\section{Statistical analysis:}

The statistical package for social sciences (SPSS) version 25.0 were used to analysis the data. statistical data, mean, and standard Error. The groups were compared using the independent sample t-test (unpaired t-test between two groups), the chi square test (for non-continuous data or percentages). Using Receiver operative characteristics (ROC) c, the cut off value, sensitivity, and specificity were determined.

\section{Result:}

The results which are lustrated in Table 1 shows the values of serum Claudin-3 (Psoriasis diagnostic marker) in the whole group of patients, the two sub-groups of patients (mild and severe - moderate) and their controls.

The concentration of serum claudin-3, was $2.18 \pm 0.61( \pm \mathrm{SE}) \mathrm{ng} / \mathrm{mL}$ (range of: 1.35-3.56) for psoriatic patients compared with the healthy controls who had $1.27 \pm .03 \mathrm{ng} / \mathrm{ml}$ (range of :1.2 -1.3 ) with a significant difference between them; $(\mathrm{P}<0.05)$.

The psoriatic patients were divided into two subgroups based on the Psoriasis Area Severity Index (PASI) They were: mild cases (PASI $<10 ; \mathrm{n}=21$ ) having serum claudin-3 of $1.69 \pm 0.08 \mathrm{ng} / \mathrm{mL}$ (range: $1.35-1.92$ ) and moderate - severe cases (PASI $\geq 10 ;(\mathrm{n}=19)$ with claudin-3 value of $2.73 \pm 0.12 \mathrm{ng} / \mathrm{mL}$ (range :2.23.56).

As in table 1, and fig. 1 and 2a, claudin-3 shows a significant difference between the PASI in the severemoderate cases and the controls $(\mathrm{p}<0.05)$, a significant positive difference between the severe- moderate cases and the mild cases $(\mathrm{p}<0.05)$ and a significant difference between the mild cases and the controls $(\mathrm{p}<$ $0.05)$.

No significant correlation was found between claudin-3 and BMI (fig 2 b).

Table 1: Mean $( \pm \mathrm{SE})$ value of Psoriasis diagnostic (Claudin-3) in the patients (with the two subgroups: mild and severe - moderate) and their controls.

\begin{tabular}{|c|c|c|}
\hline $\begin{array}{l}\text { Parameters } \\
\text { Groups }\end{array}$ & $\begin{array}{l}\text { CLDN-3 cons. } \\
(\mathrm{ng} / \mathrm{ml})\end{array}$ & range \\
\hline All Patients $(\mathrm{n}=40)$ & $2.18 \pm 0.16$ & $1.35-3.56$ \\
\hline $\begin{array}{l}\text { Severe \& Moderate } \\
\quad(\mathrm{n}=19), \text { PASI } \geq 10\end{array}$ & $\begin{array}{l}2.73 \pm 0.12 \\
\mathrm{~A}\end{array}$ & $2.2-3.56$ \\
\hline $\begin{array}{l}\text { Mild } \\
(\mathrm{n}=21), \text { PASI } \leq 10\end{array}$ & $\begin{array}{l}1.69 \pm 0.08 \\
\mathrm{~B}\end{array}$ & $1.35-1.92$ \\
\hline Controls & $1.27 \pm 0.03$ & $1.2-1.3$ \\
\hline
\end{tabular}




\begin{tabular}{l|l|r} 
& C & All \\
LSD & 0.39 & pati \\
ent
\end{tabular}

s group vs controls, $p<0.05$, LSD. Least significant difference

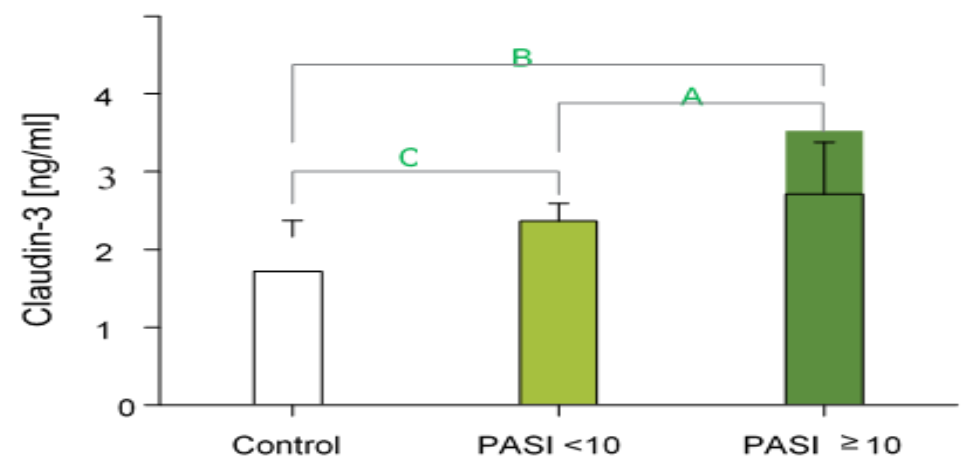

Figure 1: Serum Claudin-3 concentration according to psoriasis severity.

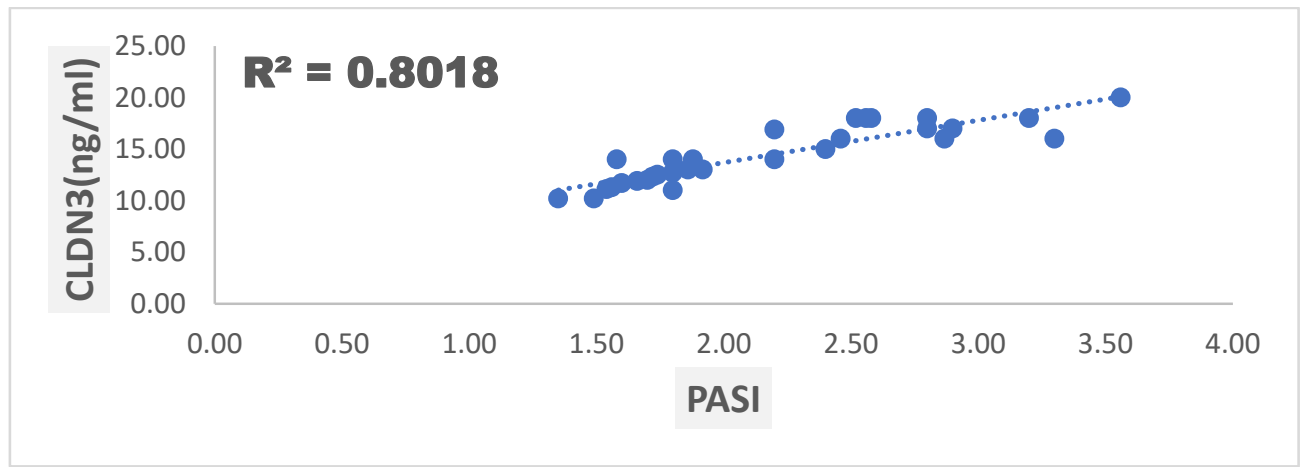

Figure 2a: Correlations of claudin-3 with PASI, Psoriasis Area Severity Index. $(p<0.001)$.

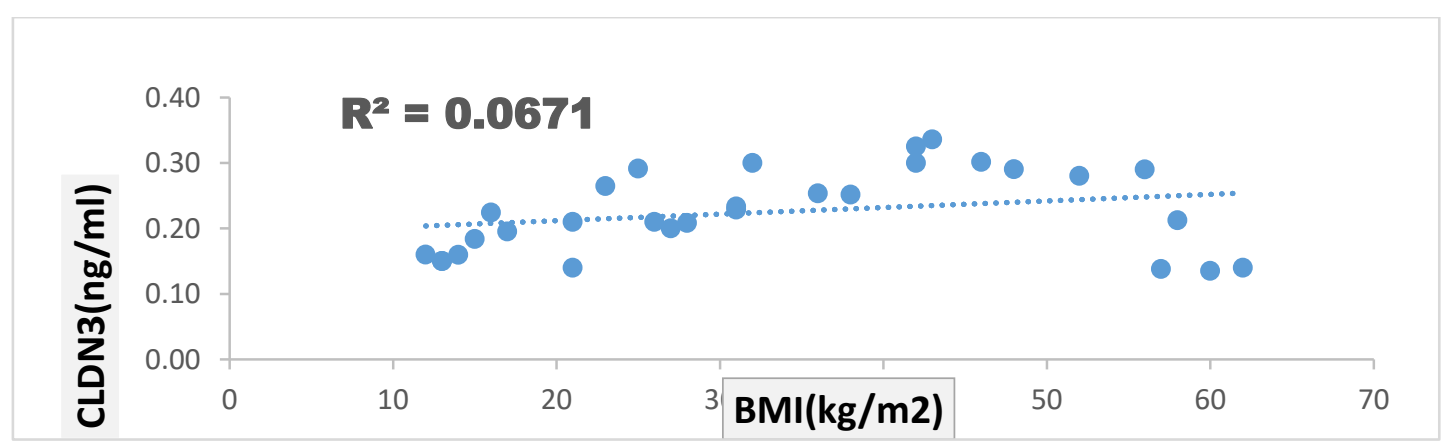

Figure 2b: correlation of claudin-3 with BMI, body mass index.

\section{Diagnostic Value of Claudin-3.}

Receiver operating characteristic (ROC) curve was used to evaluate the diagnostic value of claudin-3 in psoriasis. 
Figure 3 shows ROC for claudin-3 concentration in the context of discrimination between patients and control. The area under the curve (AUC) was $0.883, \mathrm{p}<0.001$. The sensitivity and specificity of the test at cut off value of claudin-3 concentration $>1.5 \mathrm{ng} / \mathrm{ml}$ were 73.33 and 94.74 respectively.

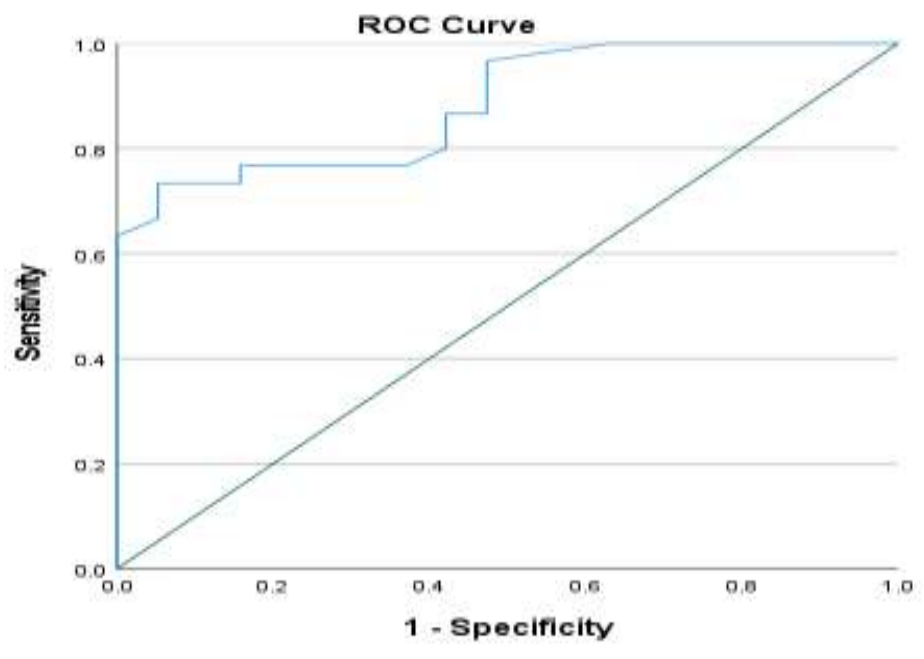

Figure 3: The ROC for Claudin-3 as a diagnostic marker for psoriasis.

Cldn3 test Cutoff value Sensitivity Specificity $\quad+$ pv $\quad-p v \quad$ Accuracy Auc

\begin{tabular}{|c|}
\hline$<0,001$ \\
\hline
\end{tabular}

$+P V$, positive predictive value; $-P V$, negative predictive value, $A U C=$ Area under curve.

\section{Demographic and Biomedical Markers of patients and control groups.}

The table 2 shows the values of age, sex and body mass for each of the patient's group and the control group,

The mean value of patients group age was not significantly different from that of the control group.

The ratio of Female to male was about the same in both groups.

The percent of smokers was about the same in both groups.

The slightly higher body mass index, BMI, in the psoriatic patients was not significantly different from that of the controls.

The entire group of patients in this study had a mean disease duration of $7.3( \pm 3.7 \mathrm{SE})$ years with a mean of Psoriasis area severity index of $12.4( \pm 5.4 \mathrm{SE})$.

Table 2: Mean $( \pm \mathrm{SE})$ Values of Demographic Parameters for the Group of Psoriatic Patients and their controls. 


\begin{tabular}{|c|c|c|c|}
\hline Parameter & $\begin{array}{l}\text { Patients } \\
n=40\end{array}$ & $\begin{array}{l}\text { Controls .... } \\
\mathbf{n = 3 0}\end{array}$ & $\begin{array}{l}\text { Static } \\
\text { significant }\end{array}$ \\
\hline Age (years) & $46.3 \pm 15.5$ & $45.6 \pm 12.1$ & NS \\
\hline Gender (F/M) & $15 / 25$ & $11 / 19$ & NS \\
\hline BMI $\left(\mathrm{Kg} / \mathbf{~ m}^{2}\right)$ & $29.3 \pm 5.4$ & $28.1 \pm 64$ & NS \\
\hline Smoking & $35 \%$ & $30 \%$ & NS \\
\hline $\begin{array}{l}\text { Disease } \\
\text { duration(years) }\end{array}$ & $7.3 \pm 3.7$ & ------ & -------- \\
\hline PASI score & $12.4 \pm 5.4$ & -------- & ------- \\
\hline
\end{tabular}

Serum lipids in the psoriatic patients and their controls.

Table 3 shows the comparison of serum levels of lipids in patients with psoriasis and the healthy control group.

There were no significant differences in the serum TC and HDLC between the two groups. Serum LDLC and TG were significantly higher in the patient's group as compared to the controls.

Table 3: Mean $( \pm$ SE) Values of lipid profile for Entire Group of Psoriatic Patients and their controls.

\begin{tabular}{|llllll}
$\begin{array}{l}\text { Parameters } \\
\text { Groups }\end{array}$ & $\begin{array}{l}\text { TC } \\
\mathbf{m g} / \mathbf{d L}\end{array}$ & $\begin{array}{l}\text { TG } \\
\mathbf{m g} / \mathbf{d L}\end{array}$ & $\begin{array}{l}\text { HDLC } \\
\mathbf{m g} / \mathbf{d L}\end{array}$ & $\begin{array}{l}\text { LDLC } \\
\mathbf{m g} / \mathbf{d L}\end{array}$ & $\begin{array}{l}\text { VLDLC } \\
\mathbf{m g} / \mathbf{d L}\end{array}$ \\
\hline $\begin{array}{c}\text { Patients } \\
\text { n = 40 }\end{array}$ & $\mathbf{1 6 3 . 9}$ & $\mathbf{1 9 0 . 2} \pm 12.3$ & $\mathbf{4 0 . 1 7}$ & $\mathbf{1 1 6 . 6 5}$ & $\mathbf{3 4 . 8 6}$ \\
& \pm 7.58 & & \pm 1.57 & \pm 6.86 & \pm 2.93 \\
Controls & $\mathbf{1 4 1 . 7}$ & $\mathbf{1 1 9 . 7}$ & $\mathbf{4 2 . 1 6}$ & $\mathbf{9 0 . 0}$ & $\mathbf{2 3 . 8 3}$ \\
n = 30 & \pm 4.87 & \pm 13.8 & \pm 3.35 & \pm 7.11 & \pm 2.93 \\
& & & & & 0.027 \\
P value & $\mathrm{NS}$ & 0.018 & $\mathrm{NS}$ & 0.042 & \\
\hline
\end{tabular}

The $p$ values refer to the significant differences between groups at $\leq 0.05$ level, Ns: Non-significant

\section{Discussion:}

The results of the present work show clearly the elevation of the transmembrane protein claudin- 3 in psoriatic patients when compared to the controls. This is in accord with Sikora et al 2019, who had, also, reported a positive correlation of this marker with PASI and neutrophil-to-lymphocyte ratio[13]. 
As diagnostic markers the ROC revealed good specificity and sensitivity for marker claudin-3 (as in fig. 3 ). accordingly, it could be said that the claudin-3 marker can be used as diagnostic as well as prognostic marker.

In the current study, the mean age of psoriatic patients was $45.6( \pm 12.1)$ years, which is close to the age found by [14] which was 41.1 years. However different reports gave different figures [15] , [16] and [17].

For the duration of the disease it was, in the present study, around 9.3 years which is close to that reported by[18], 2019. Longer durations could also be found in other reports as 12, 15, or even 20 years [19], [20] and [16] .

The other factor which is usually considered in psoriasis is obesity. The present study could not find a significant difference in the patient's BMI and the controls. This results is similar to that found by [21]. While Imafuku et al., 2016 found the obesity was being associated with 2-fold increase in the risk of psoriasis when BMI changed from $19-29$

High visceral fat was reported in psoriasis in many reports [22] Visceral fat, is considered the primary source of inflammatory cytokines in obesity. Obesity and psoriasis, in particular, are both pro-inflammatory diseases in which the adipokine balance is changed in favor of proinflammatory adipokines [23] .

The other variables found in the literature of psoriasis are serum lipids. The present findings show a significantly higher serum LDLC, VLDLC and TG in the patient's and the control groups But did not reach significance level.

The higher incidence of dyslipidemia among psoriasis patients compared to the general population were covered in details by [24], with incidence of severe hyperlipidemia in severe cases of psoriasis. This gives a support to the idea that the two diseases, hyperlipidemia and psoriasis, may share similar inflammatory pathways.

The suggested mechanism is the induction of Some cytokines: interleukin (IL)-1, IL-6, and TNF-a, which play important roles in serum lipid dysregulation. This may include alteration in the function of hepatic cells and arterial smooth muscle which will induce changes in lipoprotein composition and increase in the expression of cellular adhesion molecules. These all events will increase lipid deposition on the arterial walls. [25].

Moreover, the rates of LDL, HDL and lipoprotein (a) oxidation were reported to increase in psoriasis, and considered as an early sign of atherosclerosis in psoriasis [26].

Another important feature of psoriasis is hyperuricemia as an independent risk factor. The present results show a significantly higher serum uric acid in the patient's group when compared with the controls (table 4)

Psoriasis has been reported. among other comorbidities, to associate hyperuricemia [27], [28].

In 2014 Paolo [29] reported an incidence of $20 \%$ of hyperuricemia among the study psoriatic patients and related that to obesity, and triglycerides , But not with age and sex, disease duration or PASI score.

\section{Conclusion:}

1-The results of this study revealed a considerable increase in cloudin-3 in psoriatic patients as compared to healthy controls, as well as a highly significant correlation with the Psoriasis Area Surface Index (PASI) score. This level is proportionate to the severity of the condition.

2-The ROC test demonstrated that this marker has good specificity and sensitivity.

3-The above two points may lead to the recommendation of employing Claudin -3 in addition to clinical biological markers as an adjuvant diagnostic and prognostic marker for psoriasis. 


\section{References:}

[1] L. Huang, R. Gao, N. Yu, Y. Zhu, Y. Ding, and H. Qin, "Dysbiosis of gut microbiota was closely associated with psoriasis," Sci. China Life Sci., vol. 62, no. 6, pp. 807-815, 2019.

[2] R. S. Azfar and J. M. Gelfand, "Psoriasis and metabolic disease: epidemiology and pathophysiology," Curr. Opin. Rheumatol., vol. 20, no. 4, p. 416, 2008.

[3] M. A. Lowes, M. Suarez-Farinas, and J. G. Krueger, "Immunology of psoriasis," Annu. Rev. Immunol., vol. 32, pp. 227-255, 2014.

[4] A. G. Markov, A. Veshnyakova, M. Fromm, M. Amasheh, and S. Amasheh, "Segmental expression of claudin proteins correlates with tight junction barrier properties in rat intestine," J. Comp.

Physiol. B, vol. 180, no. 4, pp. 591-598, 2010.

[5] F. Benhadou, D. Mintoff, B. Schnebert, and H. B. Thio, "Psoriasis and microbiota: a systematic review," Diseases, vol. 6, no. 2, p. 47, 2018.

[6] S. Zeissig et al., "Changes in expression and distribution of claudin 2, 5 and 8 lead to discontinuous tight junctions and barrier dysfunction in active Crohn's disease," Gut, vol. 56, no. 1, pp. 61-72, 2007.

[7] M. P. Fink and R. L. Delude, "Epithelial barrier dysfunction: a unifying theme to explain the pathogenesis of multiple organ dysfunction at the cellular level," Crit. Care Clin., vol. 21, no. 2, pp. 177-196, 2005.

[8] A. I. Ivanov, I. C. McCall, C. A. Parkos, and A. Nusrat, "Role for actin filament turnover and a myosin II motor in cytoskeleton-driven disassembly of the epithelial apical junctional complex," Mol. Biol. Cell, vol. 15, no. 6, pp. 2639-2651, 2004.

[9] K. Turksen and T.-C. Troy, "Junctions gone bad: claudins and loss of the barrier in cancer," Biochim. Biophys. Acta (BBA)-Reviews Cancer, vol. 1816, no. 1, pp. 73-79, 2011.

[10] C. Barmeyer, M. Fromm, and J.-D. Schulzke, "Active and passive involvement of claudins in the pathophysiology of intestinal inflammatory diseases," Pflügers Arch. J. Physiol., vol. 469, no. 1, pp. 15-26, 2017.

[11] M. G. Rooks and W. S. Garrett, "Gut microbiota, metabolites and host immunity," Nat. Rev. Immunol., vol. 16, no. 6, pp. 341-352, 2016.

[12] F. Ciccia et al., "Dysbiosis and zonulin upregulation alter gut epithelial and vascular barriers in patients with ankylosing spondylitis," Ann. Rheum. Dis., vol. 76, no. 6, pp. 1123-1132, 2017.

[13] M. Sikora, M. Chrab\kaszcz, A. Waśkiel-Burnat, A. Rakowska, M. Olszewska, and L. Rudnicka, "Claudin-3--a new intestinal integrity marker in patients with psoriasis: association with disease severity," J. Eur. Acad. Dermatology Venereol., vol. 33, no. 10, pp. 1907-1912, 2019.

[14] K. R. R. Prabha, P. M. Lakshmi, P. C. Chittambalam, and A. S. Selvakumar, "Case control evaluation of serum vitamin D levels in psoriasis."

[15] S. I. Pavlov, I. I. Ivanova, and D. Gerova, "Vitamin D status in patients with psoriasis," Scr. Sci. Medica, vol. 48, no. 1, pp. 50-54, 2016.

[16] C. Mattozzi, G. Paolino, A. G. Richetta, and S. Calvieri, "Psoriasis, vitamin D and the importance of the cutaneous barrier's integrity: an update," J. Dermatol., vol. 43, no. 5, pp. 507-514, 2016. 
[17] M. A Elhelaly, A. E. A El-Ashmawy, A. A. A Haseeb, H. G Abd Allah, and H. S Abd-Alsamie, "Comparative Study of Vitamin D Level between Psoriatic Patients and Psoriatic Arthritis Patients," Egypt. J. Hosp. Med., vol. 72, no. 4, pp. 4300-4307, 2018.

[18] S. Pitukweerakul, S. Thavaraputta, S. Prachuapthunyachart, and R. Karnchanasorn, "Hypovitaminosis D is Associated with Psoriasis: A Systematic Review and Meta-Analysis," Kansas J. Med., vol. 12, no. 4, p. 103, 2019.

[19] A. Filoni, M. Vestita, M. Congedo, G. Giudice, S. Tafuri, and D. Bonamonte, "Association between psoriasis and vitamin D: duration of disease correlates with decreased vitamin D serum levels: An observational case-control study," Medicine (Baltimore)., vol. 97, no. 25, 2018.

[20] B. Bergler-Czop and L. Brzezińska-Wcisło, "Serum vitamin D level--the effect on the clinical course of psoriasis," Adv. Dermatology Allergol. Dermatologii i Alergol., vol. 33, no. 6, p. 445, 2016.

[21] M. Sikora, M. Chrablkaszcz, C. Maciejewski, A. Zaremba Michałand Waśkiel, M. Olszewska, and L. Rudnicka, "Intestinal barrier integrity in patients with plaque psoriasis," J. Dermatol., vol. 45, no. 12 , pp. 1468-1470, 2018.

[22] A. Ataseven, A. U. Bilgin, G. S. Kurtipek, and others, "The importance of neutrophil lymphocyte ratio in patients with psoriasis," Mater Sociomed, vol. 26, no. 4, pp. 231-233, 2014.

[23] L. Barrea et al., "Vitamin D and its role in psoriasis: An overview of the dermatologist and nutritionist," Rev. Endocr. Metab. Disord., vol. 18, no. 2, pp. 195-205, 2017.

[24] C. Ma, C. T. Harskamp, E. J. Armstrong, and A. W. Armstrong, "The association between psoriasis and dyslipidaemia: a systematic review," Br. J. Dermatol., vol. 168, no. 3, pp. 486-495, 2013.

[25] P. Libby, P. M. Ridker, and A. Maseri, "Inflammation and atherosclerosis," Circulation, vol. 105, no. 9, pp. 1135-1143, 2002.

[26] A. V Sorokin et al., "Association between oxidation-modified lipoproteins and coronary plaque in psoriasis: an observational cohort study," Circ. Res., vol. 123, no. 11, pp. 1244-1254, 2018.

[27] K.-H. Yu et al., "Management of gout and hyperuricemia: multidisciplinary consensus in Taiwan," Int. J. Rheum. Dis., vol. 21, no. 4, pp. 772-787, 2018.

[28] N. Tsuruta, S. Imafuku, and Y. Narisawa, "Hyperuricemia is an independent risk factor for psoriatic arthritis in psoriatic patients," J. Dermatol., vol. 44, no. 12, pp. 1349-1352, 2017.

[29] P. Gisondi, G. Targher, A. Cagalli, and G. Girolomoni, "Hyperuricemia in patients with chronic plaque psoriasis," J. Am. Acad. Dermatol., vol. 70, no. 1, pp. 127-130, 2014. 